\title{
Transposition versus Decompression Alone for Ulnar Nerve Entrapment in Cubital Tunnel
}

Randa Abd Eldayem A. Said*, Magdy El-Sayed Rashed, Hosni Hassan Salama and Ibrahim Metwally Abdel Fattah

Department of Neurosurgery, Faculty of Medicine, Zagazig University, Egypt.

*Corresponding author: Randa Abd Eldayem Said, Mobile: (+2)01092320958, Email: randaabdeldayem91@gmail.com

\begin{abstract}
Background: Cubital tunnel syndrome is the most common form of ulnar nerve entrapment (UNE). Objective: The aim of the present study was to evaluate patient-reported and surgeon-evaluated outcome of ulnar nerve entrapment in cubital tunnel decompression versus transposition.

Patients and methods: The study was conducted on 18 ulnar nerve entrapment patients 9 cases underwent simple decompression operation and 9 cases underwent transposition operation. All cases were subjected to electrodiagnostic tests. Post-operative outcomes were assessed and graded, based on patient-reported and surgeon-evaluated outcome, into four groups: cured, improved, unchanged or exacerbated.

Results: Our study showed that $88.9 \%$ of the decompression group showed full motor power (FMP) postoperatively while this was shown in $44.4 \%$ of transposition group with significant difference $(\mathrm{P}<0.05)$ between the two groups. All patients of both groups showed postoperative improved sensory manifestations. There was highly significant difference ( $\mathrm{p}<0.05)$ between the two studied groups regarding postoperative nerve conduction velocity (NCV) as it was higher in decompression group than transposition group. while there was no significant difference between them regarding nerve thickness by ultrasound.

Conclusion: Simple decompression is associated with less operative time, less amount of blood loss, smaller wound, less exposure to nerve, less need to affect elbow joint except in large deformation than surgical treatment with transposition.
\end{abstract}

Keywords: Transposition, Decompression, Carpal tunnel syndrome, Ulnar nerve entrapment.

\section{INTRODUCTION}

Cubital tunnel syndrome is the second most common upper extremity entrapment neuropathy following carpal tunnel syndrome. Symptoms progress of cubital tunnel from mild intermittent numbness induced with elbow flexion to constant anesthesia. Pain and tenderness over the medial epicondyle and cubital tunnel may be present with flexion and extension of the elbow. Weakness of ulnar nerve-innervated intrinsic hand muscles can also be seen at this time. Weakness starts with clumsiness and loss of dexterity of the hand, with progression to weakness of grip and pinch (Froment's sign) ${ }^{(\mathbf{1}, \mathbf{2})}$.

The elbow flexion test is performed with both elbows maximally flexed and wrists in full extension for three minutes, while the pressure provocative test is done by applying pressure for 60 seconds over the ulnar nerve just proximal to the cubital tunnel with the elbow flexed at $20^{\circ}$ and forearm is supinated. These tests are positive if symptoms of pain, numbness, and paresthesia occur in the ulnar nerve distribution ${ }^{(3)}$.

Many surgical procedures are advocated for the treatment of cubital tunnel syndrome, including simple decompression, anterior transposition (subcutaneous, submuscular, intramuscular), and medial epicondylectomy ${ }^{(4)}$.

Surgical treatment of patients with cubital tunnel syndrome is governed by the following principles: release all possible sites of compression; preserve the vascularity of the ulnar nerve at the elbow, allow early mobilization of the elbow, and if the nerve subluxates during surgery, then perform a medial epicondylectomy (5). Anterior transposition surgical procedures can be grouped into 3 classes, depending on the location of the transposed ulnar nerve: subcutaneous anterior transposition, submuscular anterior transposition, and intramuscular anterior transposition ${ }^{(6)}$.

Persistent symptoms may be related to an incomplete release of structures affecting the ulnar nerve or to intraneural pathology, while recurrence after primary surgery may be due to perineural scarring and adhesions. Predictors of recurrence are female gender, age $<50$ years, concomitant carpal tunnel syndrome (CTS), clinically mild ulnar nerve entrapment (i.e., McGowan stage I) and previous elbow fracture or dislocation ${ }^{(7)}$. Ulnar nerve entrapment is usually treated with ulnar nerve transposition, achieving satisfactory results in $73-82 \%$ of patients. The main improvement is reduction of pain whereas return of sensibility and motor function are more unpredictable ${ }^{(8)}$.

The aim of the present study was to evaluate patient-reported and surgeon-evaluated outcome of ulnar nerve entrapment in cubital tunnel decompression versus transposition.

\section{PATIENTS AND METHODS}

This study was anon-randomized case-control study that was conducted at Neurosurgery Department, Zagazig University Hospitals on 18 surgically-treated ulnar nerve entrapment patients. 9 cases were treated by simple decompression and 9 cases were treated by transposition. This study was done for a year. Patients 
with concomitant cervical radiculopathy and traumatic ulnar nerve injury were excluded.

\section{Ethical approval:}

The study was approved by the Ethical Committee of Zagazig Faculty of Medicine. An informed consent was obtained from all patients in this research. Every patient received an explanation for the purpose of the study. All given data were used for the current medical research only. This work has been carried out in accordance with The Code of Ethics of the World Medical Association (Declaration of Helsinki) for studies involving humans.

Clinical diagnosis and evaluation was done for all cases based on patient history, symptoms and physical examination, including Tinel's test, muscle strength in ulnar nerve-innervated muscles, ulnar nerve subluxation and present muscle atrophies in ulnar nerve-innervated muscles and Forment sign.

Electrodiagnostic tests including nerve conduction studies and needle EMG were performed bilaterally in all patients using standard techniques to confirm the diagnosis of cubital tunnel syndrome, and to determine the severity of the disease, to localize the area of compression in some cases, and to exclude other sites of compression. Simple decompression is the method of choice in ordinary cases, but if a clear luxation or hypermobility of the ulnar nerve is seen preor peri-operatively, a submuscular (or sometimes subcutaneous) nerve transposition is performed.

\section{Surgical technique:}

Patients with fixed sensory loss, pain, weakness, or significant denervation on EMG were considered for surgical treatment. Two different operative procedures were used: simple ulnar nerve decompression without epicondylectomy, and anterior transposition of the ulnar nerve (subcutaneous or intramuscular) with or without use of a microscope. Surgical intervention was performed under general anesthesia. Patients were positioned supine, and the externally rotated arm was placed on the arm-table with the elbow flexed and the forearm in the supine position.

Simple decompression was performed through a $6-10 \mathrm{~cm}$ long curved incision, slightly going ventral to the medial epicondyle avoiding exposure and scar directly over the nerve at sulcus level, along the course of the ulnar nerve. The ligament of Osbourne and the superficial and deep fascia of the flexor carpi ulnaris muscle were incised. The Struther's arcade or any aponeurosis was not released if the clinical findings did not indicate anything else. The ulnar nerve itself was then retained on its bed and not circumferentially dissected from the surrounding connective tissue.

Subcutaneous or submuscular anterior transposition of the ulnar nerve was performed in some patients with severely tensed ulnar nerve due to cubitus valgus over $30^{\circ}$ or those engaging in work using the inside of the elbow or those receiving reoperation. In addition, the decision to use a microscope was made at random. The final choice of subcutaneous or submuscular anterior transposition of the ulnar nerve depends on the surgeon and is based on patient and clinically-related factors, e.g. patient comorbidity, and amount of available fat tissue.

In some cases, the preoperative examination was supplemented with an X-ray of the elbow or MRI of the cervical spine if there was suspicion of another disorder or diagnosis. These cases were excluded as well.

\section{Outcomes:}

Postoperative outcomes were assessed and graded, based on patient-reported and surgeonevaluated outcome, into four groups: cured, improved, unchanged or exacerbated (i.e. previously defined as worsened). Following this distribution, cases were reassigned into two groups for clinical reasons: cured/improved and unchanged/exacerbated.

\section{Statistical analysis:}

The collected data were coded, processed and analyzed using the SPSS (Statistical Package for Social Sciences) version 22 for Windows ${ }^{\circledR}$ (IBM SPSS Inc, Chicago, IL, USA). Data were tested for normal distribution using the Shapiro Walk test. Qualitative data were represented as frequencies and relative percentages. Chi square test $\left(\chi^{2}\right)$ to calculate difference between two or more groups of qualitative variables. Quantitative data were expressed as mean \pm SD (standard deviation). Independent samples t-test was used to compare between two independent groups of normally distributed variables (parametric data). $\mathrm{P}$ value $\leq 0.05$ was considered significant.

\section{RESULTS}

The present study showed that the mean age of the studied group was $28.063 \pm 5.397$ years in TAP block group and $27.438 \pm 3.425$ years in control group. No significant differences between both groups regarding demographic data as shown in details in (Table 1). There were no statistical significant differences $(\mathrm{P}>0.05)$ between the two studied groups regarding the preoperative data. All patients in decompression group and most of patients in transposition group had no previous trauma to elbow. All patients in both groups had sensory symptoms like tingling and numbness. On the other hand, most of patients in decompression group (77.8\%) and near half of patients $(44.4 \%)$ in transposition group had no deformity and had full motor power. Regarding Froment test, there was no significant difference between the two studied groups $\mathrm{P}>0.05$. Moreover, the Tinel's test was positive in all cases of both groups. There was no deformity in $77.8 \%$ of decompression group and in $44 \%$ of transposition group with no significant difference between the groups (Figure 1). 
The preoperative mean of NCV was $37.48 \pm 7.1$ $\mathrm{m} / \mathrm{s}$ and $36.7 \pm 4.35 \mathrm{~m} / \mathrm{s}$ in decompression group and transposition group respectively with no significant difference between them $(\mathrm{p}<0.05)$. Also, the preoperative mean of nerve thickness by ultrasound in both groups was almost equal $(21.3 \pm 7.1$ and $21.8 \pm 5.1$ mm respectively) (Table 2 ).

The mean amount of blood loss without using tourniquet in decompression group was significantly ( $\mathrm{p}$ $<0.05)$ lower than that of transposition group $(26.67 \pm$ $7.07 \mathrm{cc}$ and $48.89 \pm 13.6 \mathrm{cc}$ respectively). Also, the mean time of operation in decompression group (46.6 \pm $3.5 \mathrm{~min}$ ) was significantly ( $<0.05$ ) lower than that of transposition group $(67.78 \pm 16.2 \mathrm{~min}) .88 .9 \%$ of the decompression group showed full motor power (FMP) postoperatively while this was shown in $44.4 \%$ of transposition group with no significant difference $(\mathrm{P}<$ 0.05 ) between the two groups. All patients of both groups showed postoperative improved sensory manifestations, while the deformity was improved postoperatively in equal percent $(22.2 \%)$ of both groups. Regarding postoperative complications (infection, hematoma, injury to vessels and joint stiffness) no one in both groups reported any complications. Regarding Froment sign post operatively, there was improvement of both group with no significant difference. Regarding Tinel's test postoperatively, all cases of both groups improved showing negative test. There was no more post-operative deformity and $22.2 \%$ of patients in both groups showed improved deformity (Figure 2).

There was highly significant $(\mathrm{p}<0.001)$ increase in nerve conduction velocity (NCV) postoperative than preoperative in both groups. Also, there was highly significant decrease in nerve thickness by (U/S) postoperative than preoperative in decompression group and this decrease in thickness was significant $(p<0.05)$ in transposition group. There was a significant difference $(p<0.05)$ between the two studied groups regarding postoperative nerve conduction velocity $(\mathrm{NCV})$ as it was higher in decompression group than in transposition group $(57.28 \pm 2.5$ vs. $54.18 \pm 3.35 \mathrm{~m} / \mathrm{s})$. While, there was no significant difference between both groups regarding nerve thickness by ultrasound (Table 3).

Table (1): Demographic data of the studied groups ( $\mathrm{n}=18$ )

\begin{tabular}{|c|c|c|c|c|c|c|}
\hline Demographic data & Decon & group $n=9$ & \multicolumn{2}{|c|}{$\begin{array}{c}\text { Transposition } \\
\text { group } n=9\end{array}$} & Test & p-value \\
\hline $\begin{array}{l}\text { Age: } \\
\text { Mean } \pm \text { SD } \\
\text { Median } \\
\text { Min-Max }\end{array}$ & \multicolumn{2}{|c|}{$\begin{array}{c}42.7 \pm 15.1 \\
45 \\
20-65\end{array}$} & \multicolumn{2}{|c|}{$\begin{array}{c}40.2 \pm 15.08 \\
44 \\
18-63\end{array}$} & 4.5 & 0.8 \\
\hline Sex: & $\mathbf{N}$ & $\%$ & $\mathbf{N}$ & $\%$ & & \\
\hline $\begin{array}{l}\text { Male } \\
\text { Female }\end{array}$ & $\begin{array}{l}8 \\
1\end{array}$ & $\begin{array}{l}88.9 \\
11.1\end{array}$ & $\begin{array}{l}7 \\
2\end{array}$ & $\begin{array}{l}77.8 \\
22.2\end{array}$ & 0.4 & 0.5 \\
\hline
\end{tabular}

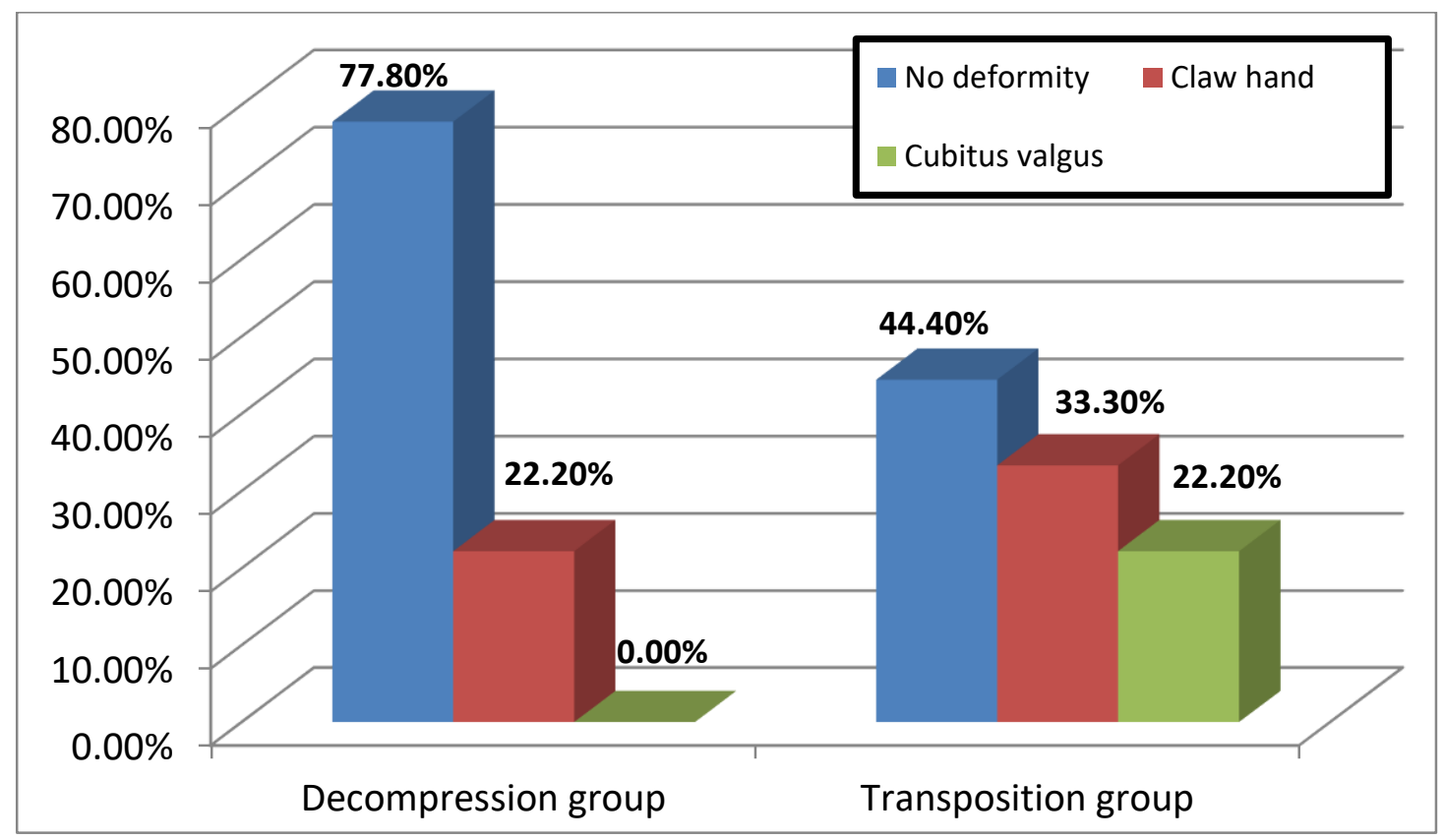

Figure (1): Comparison between the two studied groups regarding preoperative presence of deformity $(\mathrm{n}=18)$ 
Table (2): Comparison between the two studied groups regarding pre-operative nerve conduction velocity (NCV) and ulnar nerve thickness

\begin{tabular}{|l|c|c|c|c|}
\hline $\begin{array}{c}\text { Preoperative NCV and } \\
\text { thickness }\end{array}$ & Decompression group n=9 & $\begin{array}{c}\text { Transposition } \\
\text { group n=9 }\end{array}$ & t-test & p-value \\
\hline NCV(m/s): & $37.48 \pm 7.1$ & $36.74 \pm 4.35$ & 0.26 & 0.79 \\
Mean \pm SD & $30-49$ & $32-46$ & & \\
Min-Max & $21.3 \pm 7.1$ & $21.8 \pm 5.1$ & 0.19 & 0.85 \\
\hline Thickness(U/S) (mm): & $10-36$ & $10-28$ & & \\
Mean \pm SD & Min-Max & & & \\
\hline
\end{tabular}

N.B.: Normal ulnar nerve thickness in cubital tunnel $9 \mathrm{~mm}$. Normal ulnar verve NCV 50-60m/s.

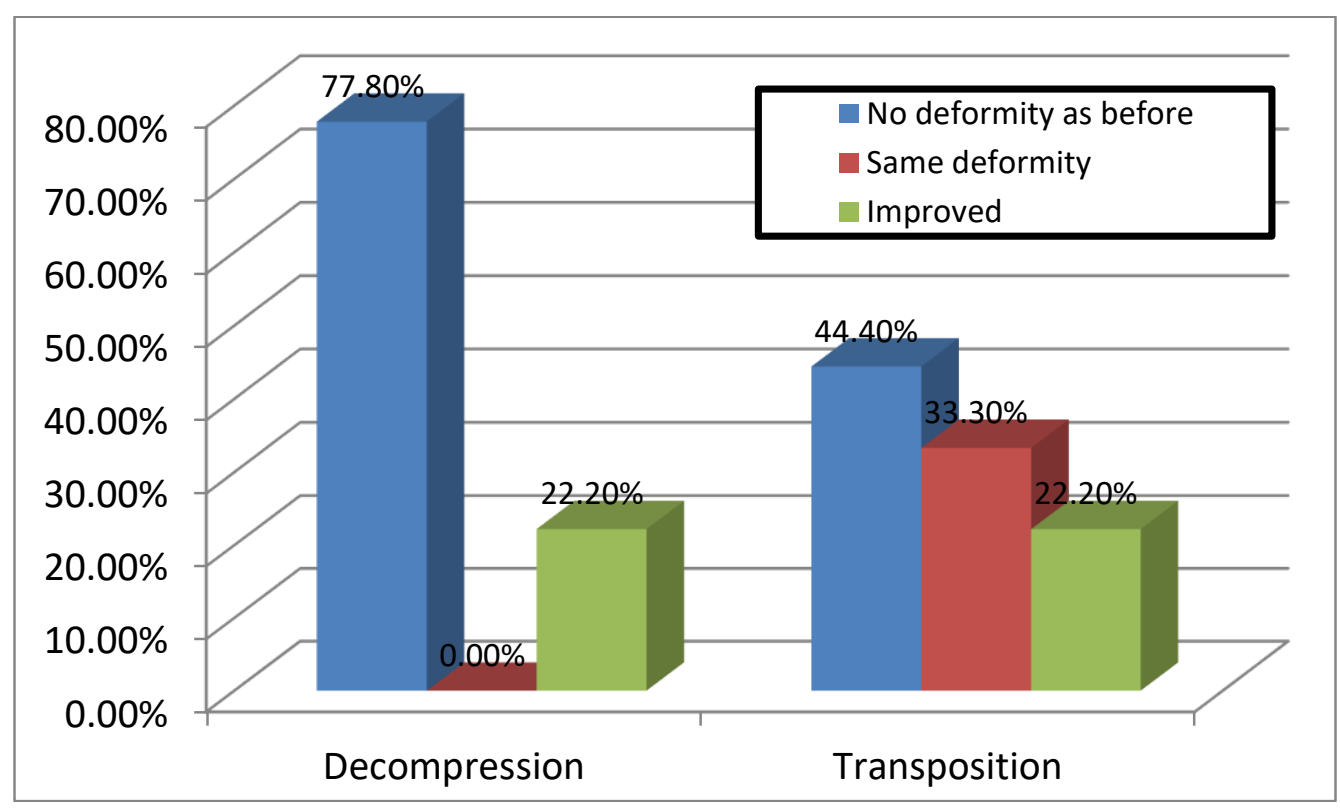

Figure (2): Comparison between the two studied groups regarding post-operative presence of deformity ( $\mathrm{n}=18)$.

Table (3): Comparison between the two studied groups regarding post-operative nerve conduction velocity (NCV) and ulnar nerve thickness $(\mathrm{n}=18)$

\begin{tabular}{|l|c|c|c|c|}
\hline \multicolumn{1}{|c|}{$\begin{array}{c}\text { Post-operative NCV and } \\
\text { thickness }\end{array}$} & $\begin{array}{c}\text { Decompression } \\
\text { group n=9 }\end{array}$ & $\begin{array}{c}\text { Transposition } \\
\text { group n=9 }\end{array}$ & t-test & p-value \\
\hline NCV(m/s): & $57.28 \pm 2.5$ & $54.18 \pm 3.35$ & 2.2 & $\mathbf{0 . 0 4 *}$ \\
Mean \pm SD & $53-60$ & $50-58$ & & \\
Min-Max & $11.1 \pm 3.2$ & $13.67 \pm 3.84$ & 1.5 & 0.14 \\
\hline Thickness(U/S) (mm): & $7-18$ & $9-19$ & \\
Mean \pm SD & Min-Max & & \\
\hline
\end{tabular}

N.B.: Normal ulnar nerve thickness in cubital tunnel $9 \mathrm{~mm}$. Normal ulnar verve NCV 50-60m/s.

\section{DISCUSSION}

Various conservative treatments and surgical techniques have been reported in the literature as efficient and feasible treatments for the cubital tunnel syndrome. The purpose of this study is to evaluate outcome of ulnar nerve decompression in cubital tunnel versus transposition.

The current study showed that there were no significant differences $(\mathrm{P}>0.05)$ between the two studied groups regarding age and sex (15 males and 3 females). Males constituted the majority of revision transposition cases. Asamoto et al. ${ }^{(9)}$ assessed the outcomes of 81 operations for the treatment of ulnar nerve entrapment at the elbow performed on 55 males

(bilateral operations in one) and 25 females. Anker $\boldsymbol{e t}$ al. ${ }^{(10)}$ studied the outcome of subcutaneous (SCT) and submuscular (SMT) ulnar nerve transpositions due to UNE. They found that among the primary transposition cases, about half were females.

The current study showed that there was no significant difference $(\mathrm{P}>0.05)$ between the two studied groups regarding occupation. The current results are not in match with Bartels and Verbeek ${ }^{(11)}$ where they reported that heavy manual occupation with 
stationary and repetitive workload may predispose to UNE. The current results also are not in match with Anker et al. ${ }^{(10)}$ who showed that majority in both transposition groups were employed in the manual, heavy, or blue-collar type of work with a repetitive workload.

In The current study, no one in both groups had history of comorbidities. Anker et al. ${ }^{(10)}$ evaluated the outcome and potential predictors for the outcome after simple decompression in UNE. One hundred seventytwo (71\%) of the cases had comorbidity with other systemic conditions or concomitant CTS. Anker et al. (10) found that five (33\%) in the SCT group and 7 (25\%) in the SMT group had concomitant CTS.

The current study showed that majority of the cases had manual and heavy type of work with no significant difference $(P>0.05)$ between the two studied groups regarding occupation. The current results agree with Anker et al. ${ }^{(10)}$ who found that majority of the cases had manual, heavy, blue-collar type of work.

The current study showed that there were no statistical significant differences $(p>0.05)$ between the two studied groups regarding the preoperative data. All patients in decompression group and most of patients in transposition group had no previous trauma to elbow, all patients in both groups had sensory symptoms like tingling and numbness. On the other hand, most of patients in decompression group (77.8\%) and near half of patients $(44.4 \%)$ in transposition group had no deformity and had full motor power. The current results agree with Anker $\boldsymbol{e t}$ al. ${ }^{\left({ }^{(10)}\right.}$ who found that one hundred seventy-eight $(74 \%)$ had developed UNE without preceding trauma to the ulnar nerve. Most cases had numbness and paresthesia as their main symptom. Anker et al. ${ }^{(10)}$ stated that twenty-one (49\%) of the cases had developed UNE without preceding trauma to the ulnar nerve. In almost all cases, numbness and paraesthesia were the main symptoms. Preoperative subluxation of the ulnar nerve was found in majority of cases $19(60 \%)$ in the primary SCT group and $22(79 \%)$ in the primary SMT group].

The current study showed that the preoperative mean of NCV was $37.48 \pm 7.1 \mathrm{~m} / \mathrm{s}$ and $36.7 \pm 4.35 \mathrm{~m} / \mathrm{s}$ in decompression group and transposition group respectively with no significant difference between them $(\mathrm{p}<0.05)$. Also, the preoperative mean of nerve thickness by ultrasound in both groups is almost equal (21.3 \pm 7.1 and $21.8 \pm 5.1 \mathrm{~mm}$ respectively). Anker $\boldsymbol{e t}$ al. (10) carried out electrophysiology. Seven (47\%) of the cases in the primary sub cutaneous transposition (SCT) group and 18 (64\%) of the primary SMT group underwent preoperative electrophysiological examination. In $3(43 \%)$ of the SCT cases, reduced conduction velocity was found along the ulnar nerve at the elbow, $1(14 \%)$ had a nerve conduction block, and $1(14 \%)$ exhibited axonal degeneration. Among the SMT cases, 6 (33\%) had reduced conduction velocity, $1(6 \%)$ had a nerve conduction block, and as many as $10(56 \%)$ displayed axonal degeneration. No electrophysiological signs of ulnar nerve affection were found in the remainder of the primary cases.

The current study showed that the general anesthesia was used for all patients in transposition group and most of patients in decompression group while local anesthesia was used for $11.1 \%$ of decompression group. Anker et al. ${ }^{(10)}$ used axillary plexus anesthesia most frequently [187 (78\%)] and general anesthesia was used in the other cases [54 $(22 \%)]$.

The current study showed that $88.9 \%$ of the decompression group showed full motor power (FMP) postoperatively, while this was shown in $44.4 \%$ of transposition group with no significant difference $(\mathrm{P}<$ $0.05)$ between the two groups. All patients of both groups showed postoperative improved sensory manifestations, while the deformity was improved postoperatively in equal percent (22.2\%) of both groups.

The current results are not in match with Anker et al. ${ }^{(10)}$ who found that in 53/242 (22\%) of the cases, no change in, or even worsened (i.e. more pronounced symptoms compared to preoperatively), symptoms were reported postoperatively by the patients. We agree with Steiner et al. ${ }^{(12)}$ who reported that simple decompression and anterior transposition produced equal outcomes. Asamoto et al. ${ }^{(9)}$ performed simple ulnar nerve decompression or anterior transposition of the ulnar nerve (subcutaneous or intramuscular) with or without the operating microscope. All patients without improvement of neurological findings had severe preoperative symptoms. Therefore, cases of poor outcome may be related to preoperative changes in the perineural vessels. Anker et al. ${ }^{(10)}$ found that 14/15 (93\%) of SCT cases and 22/28 (79\%) of SMT cases reported cured or improved.

Regarding postoperative complications (infection, hematoma, injury to vessels and joint stiffness), no one in both groups reported any complications. Asamoto et al. (9) observed no complications in the perioperative findings and procedures in cases of poor outcome. Patients with poor outcome were in a hypersensitive state, so easily suffered damage caused by mobilizing nerves or perineural vessels due to severe perioperative neurological findings.

The current study showed significant difference $(\mathrm{p}<0.05)$ between the two studied groups regarding postoperative $\mathrm{NCV}$ as it was higher in decompression group than in transposition group $(57.28 \pm 2.5$ vs. 54.18 $\pm 3.35 \mathrm{~m} / \mathrm{s}$ ), while there was no significant difference between them regarding nerve thickness by ultrasound. The current study demonstrated that there was highly significant $(p<0.001)$ increase in NCV postoperatively than preoperatively in both groups. Also, there was highly significant decrease in nerve thickness by (U/S) postoperatively than preoperatively in decompression group and this decrease in thickness was significant ( $\mathrm{p}$ $<0.05)$ in transposition group. 
The current study showed that patient with severe chronic sensory symptom and motor affection and weakness, anterior subcutaneous transposition was found better to them than just decompression. Also post-operatively, there was no complication, no hematoma or infection or nerve damage or joint stiffness. Anker et al. ${ }^{(10)}$ concluded that patients with a preoperatively electrophysiological diagnosed nerve conduction block or axonal degeneration have higher risk of not being cured or improved after simple decompression in UNE. Older patients, those with a manual profession, and constant symptoms of UNE tend to be less improved after surgery. Gokay and Bagatur ${ }^{(13)}$ reviewed mid- and long-term results of patients with cubital tunnel syndrome who underwent subcutaneous anterior transposition of the ulnar nerve. They concluded that the relatively poor results of anterior subcutaneous transposition can be attributed to the fact that this procedure is particularly preferred in chronic patients with a long duration and advanced disease with probable intrinsic nerve damage. Subcutaneous transposition of the ulnar nerve for the surgical treatment of cubital tunnel syndrome is a reliable and easy method with a low complication rate and should be preferred for its mechanical advantage in solving the nerve traction problem.

The current study showed that there was no significant deference in improvement post-operatively regarding age or occupation as the current study was done on patients their ages ranged from 18 to 65 years old and occupation vary between manual workers, carpenters, employees and housewives. All these cases showed improvement post-operatively regardless of age or occupation. Anker et al. ${ }^{(10)}$ found that patients with comorbidity with other systemic diseases, musculoskeletal conditions or concomitant CTS have a higher risk of UNE relapse and need revision of surgery. Surgeons should assess any tendency for per-operative subluxation at primary surgery for UNE, proceeding with concomitant transposition of the nerve to minimize the need for revision surgery.

\section{CONCLUSION}

Patients with cubital tunnel syndrome have cured or improved of their ulnar nerve entrapment after surgical treatment with simple decompression with less operative time, less amount of blood loss, smaller wound, less exposure to nerve, less need to affect elbow joint except in large deformation than surgical treatment with transposition. However, perioperative assessment of ulnar nerve subluxation at primary surgery for UNE should be routine and, if found, an ulnar nerve transposition should be performed in the same surgical session, to minimize the need for revision surgery.

\section{Financial support and sponsorship: Nil. Conflict of Interest: Nil.}

\section{REFERENCES}

1. Tang D, Barbour J, Davidge K et al. (2015): Nerve entrapment: update. Plast Reconstr Surg., 135 (1): 199215.

2. Calfee R, Manske P, Gelberman $R$ et al. (2010): Clinical assessment of the ulnar nerve at the elbow: reliability of instability testing and the association of hypermobility with clinical symptoms. J Bone Joint Surg Am., 92 (17): 2801-2808.

3. Buehler M, Thayer D (1988): The elbow flexion test. A clinical test for the cubital tunnel syndrome. Clin Orthop Relat Res., 233: 213-216.

4. Mitsionis G, Manoudis G, Paschos N et al. (2010): Comparative study of surgical treatment of ulnar nerve compression at the elbow. J Shoulder Elbow Surg., 19 (4): 513-519.

5. Krogue J, Aleem A, Osei D et al. (2015): Predictors of surgical revision after in situ decompression of the ulnar nerve. J Shoulder Elbow Surg., 24 (4): 634-639.

6. Matzon J, Lutsky K, Hoffler C et al. (2016): Risk factors for ulnar nerve instability resulting in transposition in patients with cubital tunnel syndrome. J Hand Surg Am., 41 (2): 180-183.

7. Gaspar M, Kane P, Putthiwara D et al. (2016): Predicting revision following in situ ulnar nerve decompression for patients with idiopathic cubital tunnel syndrome. J Hand Surg Am., 41 (3): 427-435.

8. Vogel R, Nossaman B, Rayan G (2004): Revision anterior submuscular transposition of the ulnar nerve for failed subcutaneous transposition. Br J Plast Surg., 57 (4): 311-316.

9. Asamoto S, Böker D, Jödicke A (2005): Surgical treatment for ulnar nerve entrapment at the elbow. Neurol Med Chir (Tokyo), 45 (5): 240-245.

10. Anker $I$, Zimmerman $M$, Andersson $G$ et al. (2018): Outcome and predictors in simple decompression of ulnar nerve entrapment at the elbow. $\mathbf{J}$ Hand Microsurg., 7: 24-32.

11. Bartels R, Verbeek A (2007): Risk factors for ulnar nerve compression at the elbow: a case control study. Acta Neurochir (Wien), 149 (7): 669-674.

12. Steiner H, von Haken M, Steiner-Milz H (1996): Entrapment neuropathy at the cubital tunnel: Simple decompression is the method of choice. Actaneurochir., 138: 308-313.

13. Gökay N, Bagatur A (2012): Subcutaneous anterior transposition of the ulnar nerve in cubital tunnel syndrome. Acta Orthop Traumatol Turc., 46 (4): 243-9. 